\title{
Bos taurus-indicus hybridization correlates with intralocus sexual-conflict effects of PRDM9 on male and female fertility in Holstein cattle
}

Eyal Seroussi ${ }^{1 *}$ D, Andrey Shirak', Moran Gershoni ${ }^{1}$, Ephraim Ezra² ${ }^{2}$ Daniel Jordan de Abreu Santos ${ }^{3}$, Li Ma $^{3}$ and George E. Liu ${ }^{4}$

\begin{abstract}
Background: Crossover localization during meiotic recombination is mediated by the fast-evolving zinc-finger (ZnF) domain of gene PRDM9. To study its impact on dairy cattle performance, we compared its genetic variation between the relatively small Israeli (IL) Holsteins and the North American (US) Holsteins that count millions.

Results: Initially, we analyzed the major BTA1 haplotypes present in IL Holsteins based on the 10 most telomeric SNPs of the BovineSNP50 BeadChip. Sequencing of representative haplotype carriers indicated that for all frequent haplotypes (> 6\%), the variable PRDM9 ZnF array consisted of seven tandem ZnF repeats. Two rare haplotypes (frequency $<4 \%$ ) carried an indicine PRDM9, whereas all others were variants of the taurine type. These two haplotypes included the minor SNP allele, which was perfectly linked with a previously described PRDM9 allele known to induce unique localization of recombination hotspots. One of them had a significant $(p=0.03)$ negative effect on IL sire fertility. This haplotype combined the rare minor alleles of the only SNPs with significant $(p<0.05)$ negative substitution effects on US sire fertility (SCR). Analysis of telomeric SNPs indicated general agreement of allele frequencies $(R=0.95)$ and of the substitution effects on sire fertility $(\mathrm{SCR}, R=0.6)$ between the US and IL samples. Surprisingly, the alleles that had a negative impact on male fertility had the most positive substitution effects on female fertility traits (DPR, CCR and HCR).

Conclusions: A negative genetic correlation between male and female fertility is encoded within the BTA1 telomere. Cloning the taurine PRDM9 gene, which is the common form carried by Holsteins, we encountered the infiltration of an indicine PRDM9 variant into this population. During meiosis, in heterozygous males, the indicine PRDM9 variant may induce incompatibility of recombination hotspots and male infertility. However, this variant is associated with favorable female fertility, which would explain its survival and the general negative correlation $(R=-0.3)$ observed between male and female fertility in US Holsteins. Further research is needed to explain the mechanism underlying this positive effect and to devise a methodology to unlink it from the negative effect on male fertility during breeding.
\end{abstract}

Keywords: Genomic conflict, Recombination, Dairy, Beef, Fertility, Holstein

\footnotetext{
* Correspondence: seroussi@agri.huji.ac.il

'Agricultural Research Organization (ARO), Volcani Center, Institute of Animal

Science, HaMaccabim Road, P.O.B 15159, 7528809 Rishon LeTsiyon, Israel

Full list of author information is available at the end of the article
}

(c) The Author(s). 2019 Open Access This article is distributed under the terms of the Creative Commons Attribution 4.0 International License (http://creativecommons.org/licenses/by/4.0/), which permits unrestricted use, distribution, and reproduction in any medium, provided you give appropriate credit to the original author(s) and the source, provide a link to the Creative Commons license, and indicate if changes were made. The Creative Commons Public Domain Dedication waiver (http://creativecommons.org/publicdomain/zero/1.0/) applies to the data made available in this article, unless otherwise stated. 


\section{Background}

During meiosis, genetic recombination reshuffles homologous chromosomes to produce offspring with combinations of traits that differ from those of their parents. Thus, increased recombination rate is considered to be essential for effective selection during domestication $[1,2]$, and this trait has recently drawn much attention from cattle researchers and breeders [3-8].

Among others, the genes REC8 Meiotic Recombination Protein (REC8), Ring Finger Protein 212 (RNF212) and Cyclin B1 Interacting Protein 1 (CCNB1IP1) have been implicated in driving variation in meiotic recombination rate, with PR/SET Domain 9 (PRDM9) controlling the positioning of recombination hotspots in ruminants, as in other mammals $[3,5,6,9]$. PRDM9 is annotated at the telomeric end of Bos taurus autosome 1 (BTA1) (GenBank: NP_001306826) including four major functional domains, two of which, Krüppel Associated Box (KRAB) and SSX Repression Domain (SSXRD) nuclear localization signal, are associated with transcription repression. This transcription-repression-like module is followed by a SET domain that provides methyltransferase activity and a $\mathrm{C} 2 \mathrm{H} 2$ zinc finger $(\mathrm{ZnF})$ array that binds to DNA. During meiosis, the ZnF array directs the specific binding of PRDM9 to sites across the chromosomes, and the SET domain produces H3K4me3 and H3K36me3 trimethylations to nearby histones [10]. These modifications serve to recruit the SPO11 initiator of meiotic double stranded breaks topoisomerase (SPO11) to initiate double-strand breaks by a mechanism that involves protein-protein interactions with PRDM9's transcription-repression-like module and that eventually promotes crossing over [10].

The $\mathrm{C} 2 \mathrm{H} 2 \mathrm{ZnF}$ array of PRDM9 is the fastest evolving $\mathrm{ZnF}$ in humans and other mammals [11]; this is compatible with the evident selection at the DNAbinding sites of PRDM9 [12]. This variation may promote subfertility and male sterility in hybrids, in which PRDM9 plays a complex role (reviewed by [10]). In the dairy sector, subfertility accounts for major economic losses, and dairy cattle breeding, which focuses primarily on selection for production traits, has resulted in a decline in the reproductive performance of Holstein cows [13]. Fertility issues are also predominant in male crossbreds of Bos taurus $\times$ $B$. indicus cattle. Compared to purebreds, crossbred progeny of Holstein-Friesian and indicine cattle show poorer seminal parameters, subfertility and male sterility [14]. The current bovine PRDM9 reference sequence originates from beef cattle (US Hereford cattle) and despite much interest in this gene's function in dairy cattle, there is no Holstein PRDM9 reference sequence deposited in GenBank. In this study, we describe a longer form of PRDM9 protein that is prevalent in Holstein cattle and analyze the effects of the different forms on male and female fertility.

\section{Results \\ Computerized cloning of PRDM9 of an influential Israeli (IL) Holstein sire}

To obtain the PRDM9 sequence of a representative Holstein sire (JJ, HOLISRM000000007424), we applied deep sequencing to the genome of this leading Israeli service sire. At the end of 2018, this sire was recorded in the top 20 sires for total net merit, with more than 10,000 daughters. Being a descendant of the popular US bull OBee Manfred Justice (HOUSA000122358313), this sire represents an influential blood line of Holstein cattle. Directed assembly resulted in a 13,568-bp gene (starting the count in the $5^{\prime}$ untranslated region, Table 1) covered by 2147 reads of $100 \mathrm{bp}$ each ( 16-fold coverage). As the assembly algorithm setup required a minimal match of $98 \mathrm{bp}$, all reads were of high quality, with no variation, mismatches or gaps (see BAM-format file [ENA: ERR3237582]). This assembled sequence had 99\% nucleotide sequence identity with the reference mRNA sequence of PRDM9 (GenBank: NP_001306826.2) and similarly, consisted of 10 exons all bordered by canonical splice sites (Table 1). The first nine exons were capable of encoding 383 amino acids that were all identical to those of the reference gene and comprised the transcription-repression-like module, followed by the SET domain. The last exon was capable of encoding 344 amino acids, which showed only $93 \%$ identity to their counterparts in the reference protein (Fig. 1). Hence, the fastevolving $\mathrm{ZnF}$ array encoded by this exon was the source of all variation between the dairy and beef forms of PRDM9, resulting in a longer 727-amino-acid dairy variant compared to the reference protein of 725 amino acids from beef cattle (Fig. 1).

\section{PRDM9 expression}

Length differences between the 727-amino-acid Holstein PRDM9 variant and the 725-amino-acid reference sequence were also introduced by a different splicing scheme for the last exon. As indicated in Table 1, in our build, the splice donor is the first CAG motif $5^{\prime}$ of this exon, which is followed by another identical motif used by the reference. To verify which is the actual splicing donor, we explored RNA-Seq data deposited in the Sequence Read Archive (SRA) of NCBI. Expression was detected only in the testis and using a stringent SRA BLAST search, we located 414 reads from three RNASeq submissions of the Hereford SuperBull 99,375 testis (Domino). Of these reads, 265 were assembled into a 2586-bp complete cDNA (Fig. 1; [ENA: ERR3237910] for BAM-format and [ENA: LR536714] for annotated 
Table 1 Genomic organization of the Bos taurus PRDM9 gene (using the representative Holstein sire)

\begin{tabular}{|c|c|c|c|c|}
\hline \multirow[t]{2}{*}{ Intron $^{a}$} & \multirow{2}{*}{$\begin{array}{l}\text { Exon } \\
\text { no. }\end{array}$} & \multicolumn{3}{|l|}{ Intron } \\
\hline & & size & & size \\
\hline TGAGCACTCCAATGGCC & 1 & 75 & CCCACGgtgagaggca & 406 \\
\hline attttccttagGCCAAA & 2 & 124 & CTATAGgtaacaggaa & 307 \\
\hline cttctttccagGTTTCA & 3 & 108 & AGCAAGgtgaggggcC & 4190 \\
\hline tcattttttagGTAAAC & 4 & 50 & CAGAATgtgagtattt & 1784 \\
\hline tcatgtggaagACAATG & 5 & 157 & AAGTCGgtaagagaaa & 842 \\
\hline ctccatcttagAАСТCA & 6 & 102 & АССТСТgtgagtgССС & 216 \\
\hline ctcacctccagATTGTG & 7 & 272 & TGGCTGgtgagaaaca & 576 \\
\hline ctgacactcagATCACC & 8 & 68 & GATGAGgtgagtgcag & 1145 \\
\hline ctcgaccccagGTATGT & 9 & 194 & CCAGAGgtgagcgcca & 1677 \\
\hline tcctctttcagCAGAAT & 10 & 1294 & $\begin{array}{l}\text { GAATCATAGGCCAACAAACTACATTTAGTCACAGGAGAATTACTGCAGCCACCCCATGCCTCAGCTCTAAGGGGGCCTCAGAGGAGGTCTG } \\
\text { TGACCTGTACAGTCACCAAGAGTGTGAGGAGAGACTTCCCAGGTGGTCCAGGGGCCAAGACTCCAATTCAGGGACCCATGTTCAATACCTG } \\
\text { ATTGGGGAACTAGCTCCCACATGCTGCAACTAAGACACAGTGCAGCTGAATAAATAAATACGTAAATAAATATTTAAA...+poly-A+tail? }\end{array}$ & \\
\hline
\end{tabular}

${ }^{\mathrm{a}}$ Exon and intron sizes are given in base pairs. Intron and exon sequences are written in lowercase and uppercase letters, respectively. The first and last two bases of the introns (gt and ag for donor and acceptor splice sites, respectively) are in bold type. The initiation and stop codons, and the putative polyadenylation signal (ATG, TAG, AATAA) are in bold and underlined type. Starting from the initiation codon, the genomic and transcript sizes of the PRDM9 gene as presented here were 13,557 and $2444 \mathrm{bp}$, respectively

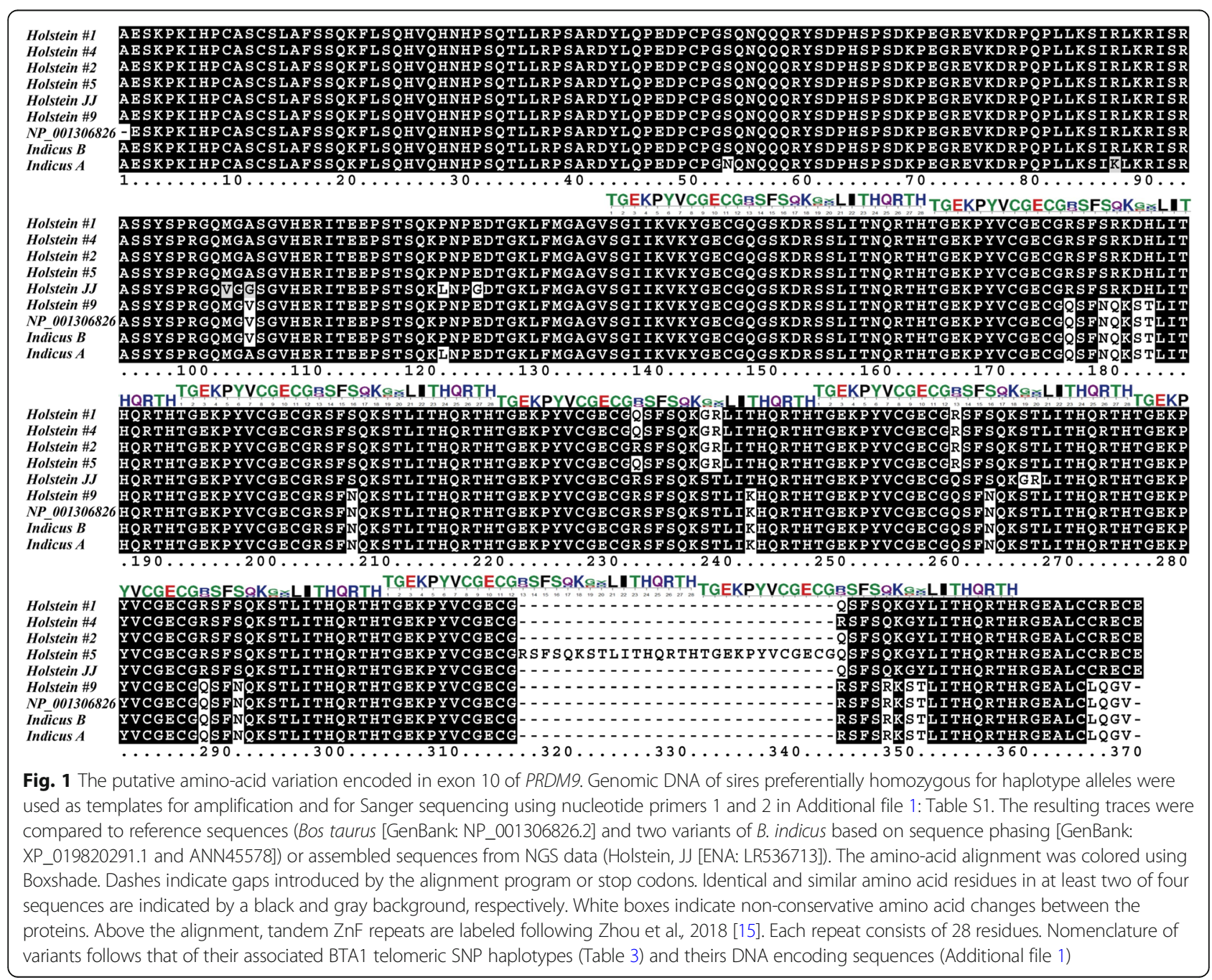


transcript sequence), which validated the first CAG motif as the donor (also exemplified by [SRA: SRR5363137.1086298]).

\section{Paralogous genes}

To further analyze variation in the Holstein PRDM9 gene, it was essential to investigate and map close paralogous sequences that may interfere with this gene's characterization. Using as template query the 13,598-bp sequence of the dairy form of PRDM9, we BLAST searched the current genome build (ARS-UCD1.2). This indicated the existence of five close paralogs (maximal score $>2500$ ): PRDM9 on BTA1 (identity 99\%, coverage 100\%), LOC100851938 on BTAX (identity $89 \%$, coverage $99 \%$ ), LOC100139638 on BTA8 (identity 92\%, coverage 99\%), LOC789895 on BTA21 (identity $82 \%$, coverage 95\%), an unannotated PRDM9-like pseudogene on BTA22 (identity 92\%, coverage 40\%) (Fig. 2). It should be noted that the latter is annotated as LOC113880961 in the hybrid cattle genome but not in the B. taurus genome build.

Diversity of the Holstein PRDM9's ZnF array was further characterized by de-novo assembly of all Holstein reads in the SRA that have been deposited by the USDA (12 bulls, [NCBI BioProject: PRJNA277147]) and that were proved similar to the reference sequence of PRDM9 exon 10 by an SRA BLAST search. This assembly resulted in five major contigs with varying lengths of $\mathrm{ZnF}$ arrays, ranging from $4 \mathrm{ZnF}$ repeats on BTA22 to over $20 \mathrm{ZnF}$ repeats on BTX, and corresponding to the five aforedescribed paralogs (Fig. 2). All reads that were assembled into the PRDM9 contig matched its dairy form of seven repeats. We used this information to design PCR primers (Additional file 1: Table S1) that would enable specific amplification, and to apply Sanger sequencing of the major variation in the $\mathrm{ZnF}$ array of Holstein PRDM9.

\section{Haplotype analysis}

Using PLINK software [16] sliding-window analysis over BTA1, we identified informative haplotypes of 10 singlenucleotide polymorphisms (SNPs) spanning the PRDM9 locus (Table 2). Scores for the male fertility trait were calculated using a linear sire model that included the insemination technician as random effect and were based on determination of pregnancy by veterinary examination for all cows that did not display estrus within $60 \mathrm{~d}$ of insemination [17]. Input data included genotypes of 1750 sires for 10 polymorphic SNPs on BTA1 that fit the Hardy-Weinberg distribution $(p<0.001)$. The PLINK permutation option was employed to verify probability of association of the haplotype alleles with male fertility. The identified haplotype consisted of the most telomeric SNPs on the BovineSNP50K BeadChip at positions 157,229, 645-157,542,408 (build ARS-UCD1.2), nearest to the PRDM9 gene $(157,545,780-157,559,387)$. For this window, 16 common haplotypes explained $>92 \%$ of the observed sequence variation (Table 3). The likelihood of association with male fertility was significant only for haplotype \#9, which associated with negative male fertility ( $\beta$ value 0.58 , Table 3$)$. This haplotype combined the rare minor alleles of the two SNPs that displayed the most negative effects on this trait (allele frequencies 3.7 and $9.1 \%$ with $\beta$ values of -0.5 and -0.3 , respectively, Table 2 ). However, since this simplified analysis may be confounded by population stratification, we applied bootstrapping with 100, 000 permutations, which corroborated the significance of this association (Table 3 ).

We further analyzed the selected haplotype using a largescale pedigree haplotyper [18]; we examined the statistically phased haplotypes and adjusted their reconstruction based on Mendelian inheritance and the complex kinship relations within the sample. The final sample for which association of the PRDM9 locus with the male fertility trait was estimated included 1414 sires with fully reconstructed and confirmed haplotypes. This analysis indicated that haplotype \#9 is associated with sires with a negative score for male fertility (chi-squared test, $p<0.05$, Table 3 ).

\section{Confirming PRDM9 association with fertility in US Holsteins}

While the association analysis is somewhat limited when using the data for the IL Holstein herd, the US population offers almost unlimited statistical power as it includes millions of individuals with Illumina BeadChip data. We used this dataset to test the association between fertility traits

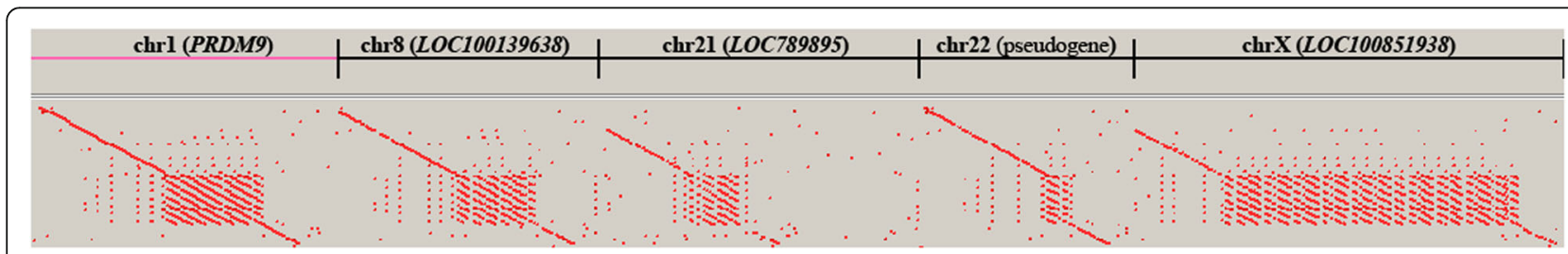

Fig. 2 PRDM9 ZnF array paralogs. Genomic reads of US Holsteins with sequence similarity to exon 10 of PRDM9 were downloaded from the SRA database and assembled using GAP5 software. Each red dot represents an 8-bp repeat that is similar to the PRDM9 exon 10 sequence. The domain of the tandem repeats forms a dotted rectangle, which reflects the number of tandem repeats 
Table 2 BTA1 telomeric SNPS

\begin{tabular}{|c|c|c|c|c|c|}
\hline SNP marker ${ }^{a}$ & BTA1 position & A1 (minor) & $\mathrm{A} 2$ & $\mathrm{MAF}^{\mathrm{b}}$ & $\beta^{c}$ \\
\hline ARS-BFGL-NGS-73542 & $157,229,645$ & A & G & 0.4020 & -0.01 \\
\hline ARS-BFGL-NGS-19721 & $157,253,652$ & G & A & 0.4090 & 0.04 \\
\hline ARS-BFGL-NGS-101788 & $157,307,208$ & A & G & 0.3740 & -0.02 \\
\hline BTA-105868-no-rs & $157,328,448$ & A & G & 0.2400 & 0.10 \\
\hline BTB-01585499 & $157,367,221$ & G & A & 0.2910 & -0.08 \\
\hline ARS-BFGL-NGS-113905 & $157,405,441$ & A & G & 0.0373 & -0.50 \\
\hline ARS-BFGL-NGS-90894 & $157,431,081$ & A & G & 0.1570 & 0.04 \\
\hline ARS-BFGL-NGS-83544 & $157,458,860$ & A & G & 0.0913 & -0.30 \\
\hline Hapmap26498-BTA-33060 & $157,503,718$ & A & G & 0.3490 & -0.05 \\
\hline ARS-BFGL-NGS-76717 & $157,542,408$ & G & A & 0.2350 & -0.16 \\
\hline
\end{tabular}

${ }^{a}$ Boldfaced names indicate SNP alleles with exceptionally low frequency and $\beta$ values

${ }^{b}$ Frequency of the minor allele was calculated based on 1750 BeadChips

c Marker effects on male fertility were estimated using PLINK Fisher's exact test

and nine BTA1 telomeric SNPs that were genotyped in both the US and IL datasets (Table 4). For these SNPs, allelic composition was very similar $(R=0.95)$ to that observed in the IL population (Table 2). Table 4 shows that all effects were significant, most of them reaching the lowest number possible by the computing software for occurrence by chance, and thus their $p$ values were indistinguishable from zero. We observed a significant correlation $(0.6)$ between the substitution effects on the male fertility trait estimated by the sire conception rate ( $\beta$ SCR values, Table 4) and the effects of these SNPs on male fertility in the IL Holstein herd ( $\beta$ values, Table 2$)$. This significant correlation indicates that the trends measured for the much smaller (2576-fold) IL substitution effects (Table 2) were also real. Indeed, for the US population as well, only the two SNPs with the lowest minor allele frequency $(\mathrm{MAF}<10 \%$, Table 4$)$, which are carried by the aforedescribed $B$. indicus haplotype, had negative effects on sire fertility (Table 4). Surprisingly, these two SNPs were the only ones with positive substitution effects on female fertility represented by heifer conception rate $(\beta$ HCR values, Table 4). As other traits of female fertility, including rates for daughter pregnancy (DPR) and cow conception (CCR), were positively correlated with HCR (Table 5), similar

Table 3 Association analysis of BTA1 telomeric SNP haplotypes with male fertility

\begin{tabular}{|c|c|c|c|c|c|c|}
\hline Haplotype $^{a}$ & $\#$ & Freq $^{b}$ & $\beta^{c}$ & STAT & $p$ & $E M P 1^{d}$ \\
\hline$G A G G A G G G G A$ & 1 & 0.2760 & 0.0749 & 0.517 & 0.472 & 0.4718 \\
\hline$A G A G G G G G A G$ & 2 & 0.1190 & -0.1120 & 0.670 & 0.413 & 0.4133 \\
\hline$A G A A A G G G G A$ & 3 & 0.0981 & 0.1570 & 0.969 & 0.325 & 0.3266 \\
\hline$A A G G G G A G G A$ & 4 & 0.0802 & 0.2560 & 2.100 & 0.148 & 0.1493 \\
\hline$G G A A A G A G G A$ & 5 & 0.0533 & -0.0120 & 0.004 & 0.952 & 0.9518 \\
\hline GAGGAGGGAG & 6 & 0.0530 & -0.2140 & 1.050 & 0.306 & 0.3071 \\
\hline$G G A A A G G G A A$ & 7 & 0.0504 & 0.3230 & 2.070 & 0.151 & 0.1498 \\
\hline GAGGAGGGAA & 8 & 0.0458 & 0.0161 & 0.005 & 0.945 & 0.9438 \\
\hline GAGGAAGAGA & 9 & 0.0370 & -0.5800 & 4.560 & 0.033 & 0.0325 \\
\hline GAGGGGGAGA & 10 & 0.0257 & 0.1530 & 0.263 & 0.608 & 0.6043 \\
\hline$G G G G A G G G A G$ & 11 & 0.0184 & 0.5370 & 2.590 & 0.108 & 0.1073 \\
\hline$A A G G A G G G A G$ & 12 & 0.0154 & -0.0296 & 0.006 & 0.937 & 0.9361 \\
\hline$A A G G A G G G G A$ & 13 & 0.0152 & 0.0878 & 0.037 & 0.847 & 0.8467 \\
\hline$A G G G A G G G G A$ & 14 & 0.0148 & -0.2660 & 0.481 & 0.488 & 0.4859 \\
\hline$A G G A A G G G A G$ & 15 & 0.0124 & -0.1210 & 0.090 & 0.764 & 0.7601 \\
\hline$A G A A G G G A G A$ & 16 & 0.0103 & 0.1830 & 0.131 & 0.717 & 0.7139 \\
\hline
\end{tabular}

a Boldfaced haplotype has a significantly low $\beta$ value

${ }^{\mathrm{b}}$ Haplotype frequency was used to sort this table

${ }^{c}$ Haplotype effects on male fertility were estimated using PLINK linear regression test

${ }^{d}$ Empirical $p$ value was the number of times the permuted haplotype-statistic exceeded $p$ in 100,000 permutations 
Table 4 Substitution effects on fertility traits in US Holstein cattle of BTA1 telomeric SNPS

\begin{tabular}{|c|c|c|c|c|c|}
\hline SNP marker ${ }^{a}$ & $\mathrm{MAF}^{\mathrm{b}}$ & $\beta \mathrm{HCR}^{c}$ & $\mathrm{P}_{\mathrm{HCR}}$ & $\beta \mathrm{SCR}^{c}$ & $P_{S C R}$ \\
\hline ARS-BFGL-NGS-73542 & 0.4158 & -0.226 & 0 & 0.015 & 0 \\
\hline ARS-BFGL-NGS-19721 & 0.3315 & -0.280 & 0 & 0.025 & 0 \\
\hline ARS-BFGL-NGS-101788 & 0.3295 & -0.250 & 0 & 0.023 & 0 \\
\hline BTA-105868-no-rs & 0.2140 & -0.126 & $1.4 \mathrm{E}-205$ & 0.004 & 5.39E-21 \\
\hline BTB-01585499 & 0.2289 & -0.315 & 0 & 0.044 & 0 \\
\hline ARS-BFGL-NGS-113905 & 0.0833 & 0.064 & $4.93 E-26$ & -0.011 & 4.35E-56 \\
\hline ARS-BFGL-NGS-90894 & 0.1095 & -0.208 & 0 & 0.034 & 0 \\
\hline ARS-BFGL-NGS-83544 & 0.0953 & 0.046 & $2.63 \mathrm{E}-15$ & -0.001 & 0.05 \\
\hline Hapmap26498-BTA-33060 & 0.2591 & -0.259 & 0 & 0.042 & 0 \\
\hline Correlation with IL values ${ }^{d}$ & 0.95 & -0.78 & & 0.60 & \\
\hline
\end{tabular}

${ }^{a}$ Boldfaced names indicate SNP alleles with exceptional frequency and $\beta$ values

${ }^{b}$ Minor alleles were the same as in Table 2 and their frequencies were calculated based on 4,508,642 BeadChips

' Marker effects on heifer and sire conception rates (HCR and SCR, with numbers of observations of 922,893 and 903,690, respectively) were estimated using PLINK Fisher's exact test

${ }^{d}$ Correlations (R) are with Table $2 \beta$ values. $R$ was -0.89 within the $\beta$ values of Table 4

effect values were also observed for these other traits (data not shown). This suggests that near the BTA1 telomere, there is a linkage between a beneficial allele that affects female fertility and an allele that reduces male fertility. These observations were supported by the moderate negative genetic correlations $(R \approx-0.3$, on average) that were generally noticed between male (SCR) and female (DPR, HCR and CCR) fertility traits in the US sample (Table 5). Consequently, strong negative correlations were observed between the effects of the BTA1 telomeric SNPs on US HCR with either IL male fertility or US SCR $(R=-0.78$ and 0.89 , respectively, Table 4).

\section{Sequence analysis of exon 10 of PRDM9 and its encoded $\mathrm{ZnF}$ array}

The rapidly evolving $\mathrm{ZnF}$ array encoded by exon 10 is thought to confer sequence specificity to the binding of PRDM9 to DNA sites in which recombination hotspots are induced. Thus, variation of this domain in heterozygotes may drive incompatibility that affects male fertility. To analyze such variation, we Sanger sequenced this $\mathrm{ZnF}$ array in a sample of individuals that were preferentially homozygous for the common haplotype alleles of the BTA1 telomeric end (haplotypes 1-10; Table 3, Fig. 1, and Additional file 1). Haplotypes \#9 and \#10 were sequenced from heterozygotes using allele-specific PCR primers
(Additional file 1: Table S1) or by subcloning into a sequencing vector. Such plasmid sequencing also enabled the identification of a PRDM9 variant with eight $\mathrm{ZnF}$ repeats, which was carried by the relatively rare haplotypes \#5 and \#7. The nucleotide sequence of this variant was virtually identical to that of the most common allele (haplotype \#1) except for an insertion in an additional sequence motif of the $\mathrm{ZnF}$ repeat (Fig. 1, [ENA: LR536717]).

Analysis of the variation of the PRDM9 $\mathrm{ZnF}$-array alleles indicated their division into two phylogenetic groups (Fig. 3). Most forms belonged to the longer 727-amino-acid dairy variant (Fig. 1), which we refer to as taurus-like type (Fig. 3). Individuals heterozygous for the rare haplotypes \#9 and \#10, which were the only haplotypes that carried the minor allele 'A' in the SNP marker ARS-BFGL-NGS-83544 ( 9\% of the population, Table 2 ), were characterized by ambiguous trace chromatograms when sequenced in the reverse orientation (Fig. 4). Such forms are compatible with the presence of the 725-amino-acid PRDM9 variant, which we refer to as the indicus-like branch (Fig. 3). This shorter form was also present in the B. taurus reference sequence and in the sequence of Dominette as assembled from trace files (data not shown), both derived from the Hereford beef breed; and in the reference sequences for B. indicus PRDM9. Hence, haplotype \#9, which was associated for sires with a negative score for male fertility, was also associated with the indicus-

Table 5 Pearson correlations between EBVs of rates of daughter pregnancy and of sire, heifer and cow conception in the US Holstein population ${ }^{\mathrm{a}}$

\begin{tabular}{llcc}
\hline & DPR & HCR & CCR \\
\hline SCR & -0.280 & -0.247 & -0.368 \\
DPR & & 0.452 & 0.880 \\
HCR & & & 0.614 \\
\hline
\end{tabular}

${ }^{a} \mathrm{DPR}$, daughter pregnancy rate; $\mathrm{SCR}, \mathrm{HCR}, \mathrm{CCR}$, sire, heifer and cow conception rates, respectively 


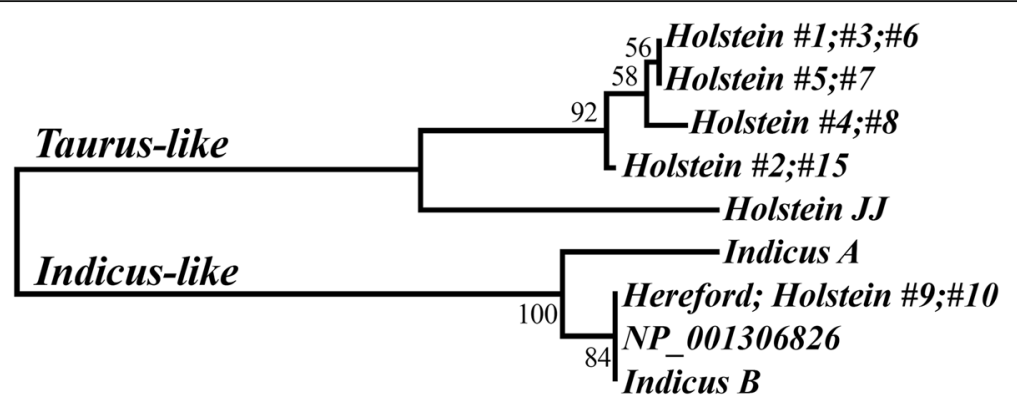

$\widehat{0.005}$

Fig. 3 Phylogenetic tree of PRDM9 ZnF array alleles. The evolutionary history of the polypeptides presented in Fig. 1 was inferred using the Neighbor-Joining method. The different alleles are identified by their carrying haplotype numbers. The optimal tree with the sum of branch length $=0.099$ is shown. Next to the branches, the percentages of replicate trees in which the associated polypeptides clustered together in the bootstrap test are shown. The tree is drawn to the scale shown in units of number of amino acid substitutions per site

like PRDM9, suggesting that it drives male infertility as in Bos taurus-indicus hybrids.

\section{Discussion}

The purpose of this study was to discover PRDM9 alleles that affect dairy production traits or could lead to more rapid genomic selection in Holstein cattle breeding by controlling the rates of meiotic recombination. On the one hand, meiotic recombination driven by PRDM9 may induce deleterious chromosomal instability and meiotic drive $[11,19,20]$; on the other, it reshuffles paternal and maternal genetic alleles in the next generation, potentially providing better novel combinations of genetic variants [15]. A recent study of US cattle showed that a specific PRDM9 allele denoted 'allele 5' has a dramatic influence on the localization of recombination hotspots and unique recombination hotspot regions that are distinguishable from hotspot regions modulated by all other alleles [15]. However, it was unclear whether this pattern would also be observed in IL Holstein cattle, which have a different demographic history, although AI using semen of elite US sires is often practiced to enhance the local variety. We applied genomic sequencing as the putative method of choice to investigate which PRDM9 alleles are prevalent in the IL Holsteins. Computerized cloning of the

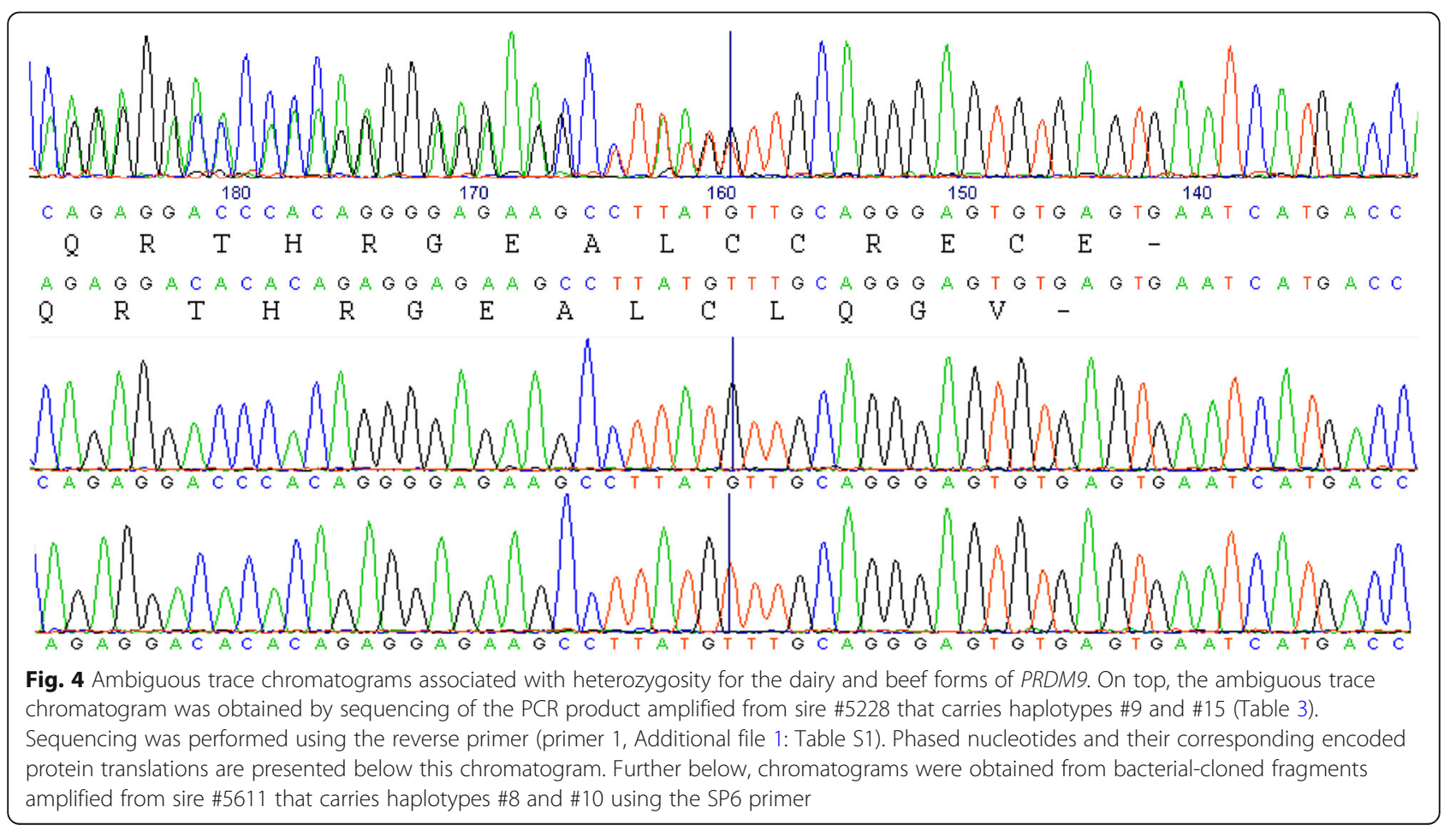


PRDM9 gene of an influential IL Holstein sire indicated that it encodes a 727-amino-acid PRDM9 variant, which we refer to as the dairy form. This form was longer than the beef form of the GenBank reference sequence, which was derived from the Hereford beef breed, as this breed's genome was the first to be sequenced, assembled, and annotated [21]. Moreover, this reference suggests an alternative splicing which does not correspond to the common transcript, as evidenced from our assembly of RNA-Seq data obtained from Hereford SuperBull 99,375 testis; as such, it should be considered a computational artifact. Based on this RNA-Seq read assembly, we give the correct transcript sequence of the beef form.

As expected [19], most of the nucleotide variation was observed within the repetitive $\mathrm{ZnF}$ array. However, in both forms, we mostly observed seven tandem $\mathrm{ZnF}$ repeats, while other repeat numbers have also been previously suggested [15]. Taking into account paralogous sequences, we carefully assembled NGS data of 12 US bulls, and concluded that all observed PRDM9 alleles have at least seven tandem $\mathrm{ZnF}$ repeats, while smaller repeat numbers belong to paralogous loci. To strengthen this conclusion, we analyzed the major BTA1 haplotypes present in IL Holsteins based on the 10 most telomeric SNPs available on the Illumina BovineSNP50 BeadChip. Sanger sequencing of representative haplotype carriers indicated that for all frequent haplotypes (frequency > 6\%), the sequences of the PRDM9 ZnF array consisted of seven tandem $\mathrm{ZnF}$ repeats. Nevertheless, two rare haplotypes (frequency $<4 \%, \# 9$ and \#10, Table 3 ) carried the beef form of PRDM9, while all others were variants of the dairy type. These two haplotypes included the minor SNP allele 'A' of rs110661033 or ARS-BFGL-NGS-83544 which was perfectly linked with allele 5 of PRDM9 [15]. Hence, these are likely to induce different localization of the recombination hotspots compared to all other haplotype alleles, as has been previously reported [15]. Moreover, haplotype $\# 9$ had a significant $(p=0.03)$ negative effect on IL sire fertility. This haplotype combined the rare minor alleles of the only SNPs tending to negative substitution effects on IL sire fertility (Table 2). To ensure this observation's significance, we analyzed nine of the most telomeric BTA1 SNPs using data from the US national dairy cattle database that includes records for millions of individuals (Table 4). This analysis indicated general agreement between the allele frequency $(R=$ 0.95 ) and the substitution effects on sire fertility (SCR, $R=0.6$ ) between the US and IL samples, confirming significant $(p<0.05)$ negative substitution effects on male fertility for both minor SNP alleles that associate with the IL \#9 haplotype that carries the beef form of PRDM9. Surprisingly, the very same alleles had the most positive substitution effects on female fertility traits
(DPR, CCR and HCR, exemplified for the latter in Table 4). This made us double-check our methodology, but realizing that "nothing in genetics makes sense except in light of genomic conflict" [22], we concluded that our results may point to a fundamental intralocus sexual conflict that arises for either the PRDM9 gene or closely linked genes at the BTA1 telomere. Such situations in which a genetic locus combines beneficial alleles for females with a selective disadvantage for males have been frequently observed (recently reviewed, [23]). This may stabilize the survival of alleles with a negative impact on fertility despite the obvious importance of this trait to genetic fitness. Indeed, in the US Holstein population, we observed a moderate negative genetic correlation between male and female fertility traits $(R \sim-0.3$, Table 5$)$; this can now be explained by the intralocus sexual conflict on the BTA1 telomere, where we recorded a much higher negative genetic correlation $(\mathrm{R} \sim 0.9)$ between the substitution effects on female and male fertilities (Table 4). Such a moderate negative correlation between male and female fertility has been observed in Danish cattle, leading to the suggestion that in breeding schemes for fertility, attention should be focused on the female side [24]. As in IL, but unlike in the US [25], male semen is not titrated according to male fertility score; it may be that the IL breeding scheme led to a much lower frequency $(<4 \%, 2.2$-fold less than in the US, Tables 2 and 4) of the minor SNP allele of ARSBFGL-NGS-113905. This allele has the highest negative impact on male fertility and thus the selection in IL against this allele decreased the negative correlation between male and female fertility traits to a non-substantial number (data not shown). It should be also noted that SCR is service sire contribution to pregnancy while HCR is the female contribution to pregnancy. Therefore, SCR is not a direct trait for male fertility, but an indirect male contribution through genetics and potentially epigenetics in sperms [26].

Our phylogenetic analysis indicated that the beef form of PRDM9 is virtually identical to $B$. indicus PRDM9. Both the taurus and indicus species descend from the extinct wild aurochs (Bos primigenius). However, separate ancient domestication events led to speciation [27] and although these species readily hybridize, male infertility is often observed in the crossbreds [14]. Low levels of haplotype sharing between $B$. taurus and indicus breeds have been frequently observed for every analyzed gene because of the recent formation of $B$. taurus $\times B$. indicus hybrids in North America [28]. This suggests infiltration into the Holstein herd of the indicine PRDM9, which induces unique recombination hotspot regions. These are not compatible with the recombination hotspots mediated by the taurine PRDM9 and thus drive meiosis in 
heterozygous individuals toward chromosomal instability and male infertility.

\section{Conclusions}

In Holstein cattle, the breeding scheme for female fertility has been complicated by a negative correlation between this trait and milk production [29]. We show that this scheme is further complicated by the negative genetic correlation between male and female fertility that is encoded in the BTA1 telomere. Cloning the taurine PRDM9 gene, which is the common form carried by Holstein haplotypes of this region, we demonstrated the infiltration of a rare indicine PRDM9 variant into the Holstein population. We suggest that during meiosis, in heterozygous males, this may induce incompatibility in the localization of recombination hotspots, destabilize genome integrity, and cause male infertility due to defects in spermiogenesis. However, the indicine PRDM9 variant was associated with a favorable effect on female fertility, which would explain the survival of this variant and the general negative correlation of $R=-0.3 \mathrm{ob}-$ served between male and female fertility traits in US Holsteins. Further research is needed to explain the mechanism underlying this positive effect on female fertility, and to devise a methodology that will unlink it from the observed negative effect on male fertility.

\section{Methods}

\section{Deep sequencing and analysis of bovine genomes}

The current reference genome is based on the Hereford beef breed. To find variations between the dairy and beef species that may underlie the differences in PRDM9, DNA was extracted from thawed frozen semen of a single Holstein sire (JJ, HOLISRM000000007424) and was deep-sequenced using the Illumina HiSeq2000 platform according to the manufacturer's paired-end protocol. Average fragment length was $580 \mathrm{bp}$, and 100-bp sequence reads were obtained from both ends. DNA sample was applied to two lanes; yielding 30-fold $(906,996$, 192 reads) coverage for this sample. The reference gene sequence was then used as a template for mapping these DNA-Seq reads using GAP5 software [30]. BWA options for this mapping were set to bam bwasw -t 8 -T 60 [31]. The assembled sequence of this sire gene was submitted under ENA accession nos. ERS3326200 (BAM format) and LR536713 (annotated gene sequence).

Additional genomic sequences of the PRDM9 locus were reconstructed using DNA-Seq reads located in NCBI's SRA and the Nucleotide BLAST tool (GenBank accession No. PRJNA277147). The reference gene sequence was then used as a template for mapping these DNA-Seq reads following the above mention procedures for assembling our own data. Further analysis of variation was performed with Sanger sequencing: DNA was amplified using PCR primers (Additional file 1: Table S1) and the Bio-X-ACT ${ }^{\mathrm{Tm}}$ Long Kit (Bioline Ltd., London, UK) according to the manufacturer's instructions under the following conditions: 30 cycles for $40 \mathrm{~s}$ at $92^{\circ} \mathrm{C}, 60 \mathrm{~s}$ at $63^{\circ} \mathrm{C}$ and $60 \mathrm{~s}$ at $68^{\circ} \mathrm{C}$. The PCR products were separated on agarose gels, excised, and purified with AccuPrep ${ }^{\oplus}$ Gel Purification Kit (BioNeer Corp., Seoul, Korea). Chromatograms were obtained by ABI3730 sequencing using a BigDye $^{\ominus}$ Terminator v1.1 Cycle Sequencing Kit (Applied Biosystems, Foster City, CA, USA). Detection and characterization of indels were performed using ShiftDetector and the ABI tracefiles [32].

\section{Cloning of PRDM9 exon10 sequence}

PRDM9 DNA fragments were amplified with subcloning primers (Additional file 1: Table S1) using Hy-Fy High Fidelity Mix (Hy Laboratories Ltd., Rehovot, Israel). The amplified products were digested by restriction enzymes, purified from a $1 \%$ agarose gel by Gel/PCR DNA Fragments Kit (Geneaid Biotech Ltd., Taipei, Taiwan) and ligated into the pGEM-T Easy Vector (Promega, Madison, WI, USA) using EcoRI and NcoI sites and T4 DNA ligase (Promega). These cloned DNA fragments were subjected to Sanger dideoxy sequencing using primers for SP6 and T7 promoters in pGEM-T Easy and an additional primer within the insert (Additional file 1: Table S1).

\section{The dataset, haplotype phasing and trait-association analysis}

Using Illumina (San Diego, CA, USA) BovineSNP50 BeadChip genotypes, four traits were analyzed: cow, heifer and daughter fertilities (CCR, HCR and DPR, respectively), and sire conception rate (IL-SCR) as previously described [33, 34]. Briefly, IL-SCR was calculated based on a linear model and 5,658,632 insemination records of 1597 sires with a minimum 250 inseminations per sire delivered by a qualified inseminator with a minimum 250 inseminations per year. Fixed effects were insemination number, AI institute, geographical region, and calendar month. Analysis of cows also included the fixed effects of parity, calving status, and day in milk at insemination. Random effects included in the model were herd-year season, insemination technician, sire of cow, and service sire. The standard deviation for IL-SCR evaluations was $0 \pm 0.024$ and mean reliability was $78.2 \%$. DNA was extracted from the semen of 1750 Holstein bulls used for AI in Israel. The bulls' identity, relationship and genetic breeding values are available at http://www.icba-israel.com/cgi-bin/bulls/en/ bl_main.htm. The dataset of IL sires, including genotyping data and SCR values is available in Excel format (Additional file 2).

Association for BTA1 SNPs was determined using PLINK [16], activating the haplotype sliding-window and bootstrapping options (--hap-window 10 --hap-linear 
--mperm 100,000). Haplotype spanning of the PRDM9 gene, consisting of 10 SNPs within positions 157,229, 645-157,542,408 (build ARS-UCD1.2), was chosen for further analyses. For this haplotype, phasing was corroborated using the rule-based Large-Scale Pedigree Haplotyper (LSPH) software [18]. The genetic correlations between traits or between markers' substitution effects were estimated as Pearson's correlation coefficients. These coefficients of correlation were calculated using $\mathrm{R}$ package [35] or CORREL function in Excel spreadsheet (Microsoft Corporation, Santa Rosa, CA, USA), respectively.

\section{US Holstein samples and analysis}

The data used were part of the 2018 US genomic evaluations from the Council on Dairy Cattle Breeding (CDCB), consisting of 1,953,934 Holstein cattle from the national dairy cattle database. Estimated breeding values (EBVs) of four fertility traits were analyzed: SCR, DPR, HCR and CCR. We only included those animals with both available genotype and trait reliability larger than the parent average. A detailed description of the data is provided in Table 6.

The genotype data from different SNP arrays were imputed to a common dataset of 4340 SNPs on BTA1 using FindHap version 3 [36]. Then, nine telomeric SNPs were analyzed: ARS-BFGL-NGS-73542, ARS-BFGL-NGS19721, ARS-BFGL-NGS-101788, BTA-105868-no-rs, BTB-01585499, ARS-BFGL-NGS-113905, ARS-BFGLNGS-90894, ARS-BFGL-NGS-83544, and Hapmap26498BTA-33060. The association studies were performed using PLINK v 1.07 software [16]. Following Garrick et al., 2009 [37], association analysis was also performed using deregressed EBVs (dEBVs) and removing the parent effect from the individual's EBV. The substitution effects estimated based on dEBVs were highly correlated with those obtained using EBVs $(R=0.956$, data not shown).

\section{Analysis of evolutionary relationships}

The evolutionary history of the PRDM9 ZnF-array alleles was inferred using the Neighbor-Joining method. Evolutionary analyses were conducted in MEGA6 [38]. Briefly, the best model was selected according to the lowest Bayesian Information Criterion (BIC) scores. The optimal tree was identified by the bootstrap test (1000 replicates). The evolutionary distances were computed using

Table 6 Description of number of animals, estimated breeding value summary statistics and average of their reliability

\begin{tabular}{llllll}
\hline Trait & $\mathrm{N}$ & Mean \pm SD & Min & Max & Reliability \\
\hline SCR & 903,690 & $2.85 \pm 0.25$ & 1.79 & 5.01 & 0.38 \\
DPR & 836,623 & $-14.41 \pm 2.73$ & -30.39 & 7.24 & 0.33 \\
HCR & 922,913 & $-0.62 \pm 2.30$ & -16.2 & 11.2 & 0.28 \\
CCR & 794,362 & $-12.22 \pm 3.42$ & -32.6 & 7.34 & 0.32 \\
\hline
\end{tabular}

the JTT matrix-based method in units of number of amino acid substitutions per site. The rate variation among sites was modeled with a gamma distribution (shape parameter $=2.53$ ). All positions containing gaps and missing data were eliminated. There were a total of 342 positions in the final dataset.

\section{Additional files}

Additional file 1: Table S1. Primer pairs used for PCR amplification and sequencing of the PRDM9 gene; and nucleotide sequences of PRDM9 exon10, partial coding sequences associated with the haplotype and individuals studied. (PDF $362 \mathrm{~kb}$ )

Additional file 2: The dataset of IL sires, including genotyping data and SCR values. (XLSX $218 \mathrm{~kb})$

\section{Abbreviations}

Al: Artificial insemination; BIC: Bayesian information criterion; CCR: Cow conception rate; CDS: Coding sequence; DPR: Daughter pregnancy rate; HCR: Heifer conception rate; NGS: Next generation sequencing; SCR: Sire conception rate

\section{Acknowledgements}

We thank Dr. Joel I. Weller for useful discussion. Contribution from the Agricultural Research Organization, Institute of Animal Science, Bet Dagan, Israel, is acknowledged.

\section{Authors' contributions}

LM, GEL (US side) and ES (Israeli side) conceived and designed the experiments. EE collected samples and generated data. AS carried out the Sanger dideoxy sequencing and haplotype analyses. ES performed the bioinformatics analyses of the DNA- and RNA-Seq data. DJDAS and MG performed computational and statistical analyses on the US and IL sides, respectively. ES drafted the paper. All authors read and approved the final manuscript.

\section{Funding}

This work was supported by BARD grant number US-4997-17 from the USIsrael Binational Agricultural Research and Development (BARD) Fund. The funders played no role in the study design, collection, analysis or interpretation of the data, writing of the manuscript, or in the decision to submit the manuscript for publication.

\section{Availability of data and materials}

Sequence data have been submitted to ENA under accession no. PRJEB31626. The dataset of IL sires, including genotyping data and SCR values, are presented in Additional file 2.

\section{Ethics approval and consent to participate}

Not applicable.

\section{Consent for publication}

Not applicable.

\section{Competing interests}

The authors declare that they have no competing interests.

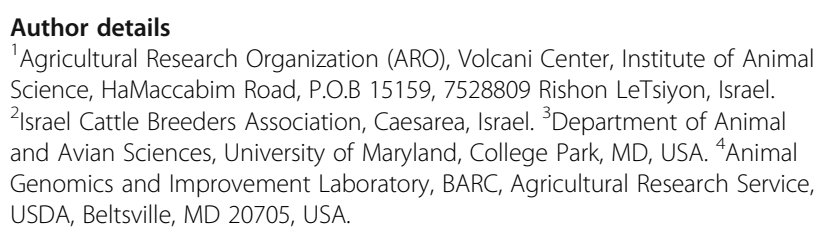


Received: 12 April 2019 Accepted: 21 August 2019

Published online: 28 August 2019

\section{References}

1. Ross-lbarra J. The evolution of recombination under domestication: a test of two hypotheses. Am Nat. 2004;163(1):105-12.

2. Ritz KR, Noor MAF, Singh ND. Variation in recombination rate: adaptive or not? Trends Genet. 2017;33(5):364-74.

3. Sandor C, Li W, Coppieters W, Druet T, Charlier C, Georges M. Genetic variants in REC8, RNF212, and PRDM9 influence male recombination in cattle. PLoS Genet. 2012;8(7):e1002854.

4. Weng ZQ, Saatchi M, Schnabel RD, Taylor JF, Garrick DJ. Recombination locations and rates in beef cattle assessed from parent-offspring pairs. Genet Sel Evol. 2014;46:34.

5. Ma L, O'Connell JR, VanRaden PM, Shen B, Padhi A, Sun C, et al. Cattle sexspecific recombination and genetic control from a large pedigree analysis. PLoS Genet. 2015;11(11):e1005387.

6. Kadri NK, Harland C, Faux P, Cambisano N, Karim L, Coppieters W, et al. Coding and noncoding variants in HFM1, MLH3, MSH4, MSH5, RNF212, and RNF212B affect recombination rate in cattle. Genome Res. 2016;26(10):1323-32.

7. Ahlawat S, De S, Sharma P, Sharma R, Arora R, Kataria RS, et al. Evolutionary dynamics of meiotic recombination hotspots regulator PRDM9 in bovids. Mol Gen Genomics. 2016;292(1):117-31.

8. Shen B, Jiang J, Seroussi E, Liu GE, Ma L. Characterization of recombination features and the genetic basis in multiple cattle breeds. BMC Genomics. 2018;19(1):304

9. Petit M, Astruc JM, Sarry J, Drouilhet L, Fabre S, Moreno CR, et al. Variation in recombination rate and its genetic determinism in sheep populations. Genetics. 2017;207(2):767-84.

10. Paigen K, Petkov PM. PRDM9 and its role in genetic recombination. Trends Genet. 2018;34(4):291-300.

11. Myers S, Bowden R, Tumian A, Bontrop RE, Freeman C, MacFie TS, et al. Drive against hotspot motifs in primates implicates the PRDM9 gene in meiotic recombination. Science. 2010;327(5967):876-9.

12. Baker Z, Schumer M, Haba Y, Bashkirova L, Holland C, Rosenthal GG, et al. Repeated losses of PRDM9-directed recombination despite the conservation of PRDM9 across vertebrates. eLife. 2017;6:e24133.

13. Ma L, Cole JB, Da Y, VanRaden PM. Symposium review: genetics, genomewide association study, and genetic improvement of dairy fertility traits. J Dairy Sci. 2018;102(4):3735-43.

14. Mukhopadhyay CS, Gupta AK, Yadav BR, Khate K, Raina VS, Mohanty TK, et al. Subfertility in males: an important cause of bull disposal in bovines. Asian-Aust J Anim Sci. 2010;23(4):450-5.

15. Zhou Y, Shen B, Jiang J, Padhi A, Park KE, Oswalt A, et al. Construction of PRDM9 allele-specific recombination maps in cattle using large-scale pedigree analysis and genome-wide single sperm genomics. DNA Res. 2018;25(2):183-94.

16. Purcell $S$, Neale B, Todd-Brown $K$, Thomas $L$, Ferreira MAR, Bender D, et al. PLINK: a tool set for whole-genome association and population-based linkage analyses. Am J Hum Genet. 2007;81(3):559-75.

17. Weller I, Ron M. Genetic analysis of fertility traits in Israeli Holsteins by linear and threshold models. J Dairy Sci. 1992;75(9):2541-8.

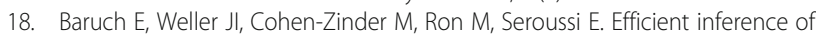
haplotypes from genotypes on a large animal pedigree. Genetics. 2006; 172(3):1757-65.

19. Berg IL, Neumann R, Lam KW, Sarbajna S, Odenthal-Hesse L, May CA, et al. PRDM9 variation strongly influences recombination hot-spot activity and meiotic instability in humans. Nat Genet. 2010;42(10):859-63.

20. Grey C, Baudat F, de Massy B. PRDM9, a driver of the genetic map. PLoS Genet. 2018;14(8):e1007479.

21. Elsik CG, Tellam RL, Worley KC, Gibbs RA, Abatepaulo ARR, Abbey CA, et al. The genome sequence of taurine cattle: a window to ruminant biology and evolution. Science. 2009;324(5926):522-8.

22. Rice WR. Nothing in genetics makes sense except in light of genomic conflict. Annu Rev Ecol Evol Syst. 2013;44:217-37.

23. Schenkel MA, Pen I, Beukeboom LW, Billeter JC. Making sense of intralocus and interlocus sexual conflict. Ecol Evol. 2018;8(24):13035-50.

24. Hansen M. Genetic investigations on male and female fertility in cattle. Livest Prod Sci. 1979;6(4):325-34.

25. Taylor JF, Schnabel RD, Sutovsky P. Review: genomics of bull fertility Animal. 2018;12(s1):s172-83.
26. Fang L, Jiang J, Li B, Zhou Y, Freebern E, Vanraden PM, et al. Genetic and epigenetic architecture of paternal origin contribute to gestation length in cattle. Commun Biol. 2019;2:100.

27. Pitt D, Sevane N, Nicolazzi EL, MacHugh DE, Park SDE, Colli L, et al. Domestication of cattle: two or three events? Evol Appl. 2019;12(1):123-36.

28. Seabury CM, Seabury PM, Decker JE, Schnabel RD, Taylor JF, Womack JE. Diversity and evolution of 11 innate immune genes in Bos taurus taurus and Bos taurus indicus cattle. Proc Natl Acad Sci U S A. 2009;107(1):151-6.

29. Wathes DC, Fenwick M, Cheng Z, Bourne N, Llewellyn S, Morris DG, et al. Influence of negative energy balance on cyclicity and fertility in the high producing dairy cow. Theriogenology. 2007;68 Suppl 1:S232-41.

30. Bonfield JK, Whitwham A. Gap5--editing the billion fragment sequence assembly. Bioinformatics. 2010;26(14):1699-703.

31. Li H, Durbin R. Fast and accurate long-read alignment with burrowswheeler transform. Bioinformatics. 2010;26(5):589-95.

32. Seroussi E, Ron M, Kedra D. ShiftDetector: detection of shift mutations. Bioinformatics. 2002;18(8):1137-8.

33. Weller Jl, Ezra E. Genetic analysis of the Israeli Holstein dairy cattle population for production and nonproduction traits with a multitrait animal model. J Dairy Sci. 2004;87(5):1519-27.

34. Seroussi E, Klompus S, Silanikove M, Krifucks O, Shapiro F, Gertler A, et al. Nonbactericidal secreted phospholipase A2s are potential anti-inflammatory factors in the mammary gland. Immunogenetics. 2013;65(12):861-71.

35. R Core Team. R: a language and environment for statistical computing. R Foundation for statistical computing. Vienna; 2013. http://www.Rproject.org/.

36. VanRaden PM, O'Connell JR, Wiggans GR, Weigel KA. Genomic evaluations with many more genotypes. Genet Sel Evol. 2011:43:10.

37. Garrick DJ, Taylor JF, Fernando RL. Deregressing estimated breeding values and weighting information for genomic regression analyses. Genet Sel Evol. 2009;41:55.

38. Tamura K, Stecher G, Peterson D, Filipski A, Kumar S. MEGA6: molecular evolutionary genetics analysis version 6.0. Mol Biol Evol. 2013;30(12):2725-9.

\section{Publisher's Note}

Springer Nature remains neutral with regard to jurisdictional claims in published maps and institutional affiliations.

Ready to submit your research? Choose BMC and benefit from:

- fast, convenient online submission

- thorough peer review by experienced researchers in your field

- rapid publication on acceptance

- support for research data, including large and complex data types

- gold Open Access which fosters wider collaboration and increased citations

- maximum visibility for your research: over $100 \mathrm{M}$ website views per year

At $\mathrm{BMC}$, research is always in progress.

Learn more biomedcentral.com/submissions 